\title{
Describing How School-Based SLPs Determine Eligibility for Children with Speech Sound Disorders
}

\author{
Kelly Farquharson, Ph.D., CCC-SLP ${ }^{1}$ and Sherine R. Tambyraja, Ph.D. ${ }^{2}$
}

The purpose of this study was to explore how school-based speech-language pathologists (SLPs) determine eligibility for children with speech sound disorders (SSDs). Presently, there is substantial variability nationwide with respect to if or how children with SSDs receive speech therapy in public schools. We report the results of a nation-wide survey of school-based SLPs, which further underscore this variability. Findings provide insight into which and how many factors SLPs report contributing to eligibility decisions, as well as which and how many components of an assessment are mandatory. Our discussion includes a call to advocacy for SLPs, but also a need for increased awareness of this problematic variability for school administrators.

KEYWORDS: speech sound disorders, IDEA, school-based practice, assessment

Learning Outcomes: As a result of this activity, the reader will be able to (1) discuss issues related to inconsistent eligibility criteria for children with speech sound disorders; (2) critique their own state/school's eligibility criteria to ensure it accommodates a range of speech sound disorders; (3) implement assessment practices that include a functional assessment of children's speech sound production skills in the classroom; (4) summarize the ways in which consistent practices are helpful to provide equal and fair services to children with speech sound disorders.

There is substantial variability in the type and amount of services provided to children with speech and language impairments in the public schools. ${ }^{1}$ In particular, service provision for children with speech sound disorders (SSDs) has frequently been reported as eclectic in
${ }^{1}$ School of Communication Science and Disorders, Florida State University, Tallahassee, Florida; ${ }^{2}$ Crane Center for Early Childhood Research and Policy, The Ohio State University, Columbus, Ohio.

Address for correspondence: Kelly Farquharson, Ph.D., CCC-SLP, 201 W. Bloxham Street, Tallahassee, FL 32301 (e-mail: kyfarq@gmail.com).
Articulation and Phonological Disorders; Guest Editor, Kathryn Cabbage, Ph.D., CCC-SLP.

Semin Speech Lang 2019;40:105-112. Copyright (C) 2019 by Thieme Medical Publishers, Inc., 333 Seventh Avenue, New York, NY 10001, USA. Tel: +1(212) 5844662.

DOI: https://doi.org/10.1055/s-0039-1677761.

ISSN 0734-0478. 
nature $^{2}$ with some inconsistencies seen across terminology, type of service delivery, ${ }^{3}$ language used for goal writing, ${ }^{4}$ and eligibility criteria. ${ }^{5}$ While all of these inconsistencies have the potential to be problematic, this is particularly true for eligibility criteria. Eligibility criteria are broadly determined at the federal level, but states and local education agencies have the autonomy to further specify guidelines. As a result, eligibility criteria may vary widely within and across states. For children with SSDs, this may result in delayed therapy services, or no therapy services at all. The aim of the present investigation is to explore the factors that speech-language pathologists (SLPs) are required to consider when determining eligibility for SSDs.

\section{SPECIAL EDUCATION SERVICES IN PUBLIC SCHOOLS}

The Individuals with Disabilities Education Act (IDEA) states that children are eligible to receive special education services if they meet three criteria: (1) the child is diagnosed with a qualifying disability; (2) the disability "adversely affects a child's educational performance"; and (3) specialized instruction and related services are necessary for the child to make progress. ${ }^{6}$ However, in a law review, Thomas ${ }^{7}$ indicated that there is no clear definition of "adversely affects" or "educational performance." Indeed, previous work suggests considerable variability within and between states with respect to which factors are considered to be related to educational performance. ${ }^{5}$ Further, Thomas ${ }^{7}$ stated that there is a difference between "educational performance" and "academic performance." Specifically, "educational performance" refers to a child's entire experience at school-not just the grades received. That is, "educational performance" considers children's social emotional well-being, their willingness to participate in class, and teacher, peer, and selfreactions to their abilities. For children with SSDs, it may be the case that their grades represent adequate attainment of curricular content. For this reason, despite having an SSD, many of these children are considered ineligible for services. However, this population of children often experiences deficits in reading, spelling, and social-emotional well-being. ${ }^{8-15}$
Thus, eligibility for services must take into consideration factors such as the connections between SSDs and reading ${ }^{16}$ and the social implications of an SSD. Further, IDEA is explicit that eligibility is a team decision and that the team includes qualified professionals (e.g., classroom teacher, SLP) and the child's parent(s) (§ 614, b, 4, A).

\section{FACTORS TO CONSIDER WHEN DETERMINING ELIGIBILITY FOR CHILDREN WITH SPEECH SOUND DISORDERS}

Although the federal guidelines may be clear, the implementation of these guidelines within and across states can be quite variable, particularly for eligibility criteria. ${ }^{5}$ Indeed, the extent to which a child's SSD impacts his/her ability to participate in the classroom will vary. However, that piece of data is crucial to determining if there is an adverse effect on educational performance. Extant research highlights the frequent use of standardized test scores as a primary means of determining eligibility. ${ }^{17}$ Although standardized testing allows for a relational analysis of children's speech, the decontextualized nature of administration as well as long-reported issues with cut-points ${ }^{18}$ can decrease validity of test results. In addition, standardized testing for SSDs is circumscribed to single-word articulation tests, ${ }^{19,20}$ which rarely include the opportunity for phonological analysis ${ }^{19}$ and very rarely provide the opportunity to rate or discuss the child's intelligibility. Further, in a review of 30 standardized speech sound assessments, McLeod and Verdon ${ }^{20}$ reported that only two include sensitivity and specificity information, which is diagnostically problematic.

In addition to basing eligibility on standardized test scores, SLPs may also be required to utilize additional criteria to determine the extent to which a child's SSD adversely affects educational performance. For example, Ireland and Conrad $^{1}$ stated that "data from teachers, parents, and the student are necessary to examine not only the student's strengths and weaknesses, but the impact on the student's education and their need for specially designed instruction". ${ }^{1(\mathrm{p} .82)}$ At present, the extent to which SLPs obtain data from teachers, parents, and the child is largely 
unknown, ${ }^{17}$ as is the weight with which SLPs consider those data as necessary for their eligibility decisions. However, data from these individuals, and direct commentary from the child, are necessary to fully form a profile regarding how the SSD is interfering with communication, attracting adverse attention, or impacting educational performance.

\section{ASSESSMENT MEASURES USED TO MAKE ELIGIBILITY DECISIONS}

SLPs typically have the autonomy to extend their assessment practices beyond one standardized test to include more functional and contextualized assessments of speech sound production (e.g., speech sample analysis, intelligibility ratings, observations). Ireland and Conrad $^{1}$ indicated that several state regulations explicitly include the use of nonstandardized measures to comprehensively capture the nature of communication impairment. Despite this, the vast majority of SLPs indicate using a single-word test, whereas shockingly few report always using a connected speech sample as a source of assessment data. ${ }^{16}$ However, connected speech samples provide rich and imperative information regarding the complexity of the SSD. ${ }^{19}$ Speech samples also afford clinicians the opportunity to examine intelligibility for each individual case. Although it can be assumed that these assessment practices influence decision making regarding eligibility for services, that remains unknown.

\section{PRESENT STUDY}

In the present study, we sought to describe current practices for determining eligibility for services for children with SSDs. Specifically, we asked the following research questions:

1. What are the factors that SLPs consider when determining eligibility for services (e.g., standardized test scores, intelligibility ratings, whether the SSD attracts adverse attention)?

2. What are the criteria that SLPs are required to use to determine eligibility for services for children with SSDs (e.g., number of standard scores and cut-points for each)?
3. Do SLPs agree with the eligibility criteria used in their school? Are there instances in which school-based SLPs believe that children with SSDs should receive services, but the children do not qualify?

4. What assessment measures are used to inform eligibility decisions?

\section{METHOD}

Data for the current investigation were obtained from a larger 53-question, web-based survey aimed at examining school-based practices for children with SSDs. The survey was distributed within the United States to school-based SLPs; there were respondents from all 50 states. Participating SLPs worked in a public school in the United States, with children, kindergarten through 12th grade. For the present study, survey questions of interest include those that explore eligibility and dismissal criteria for children with SSDs. Comprehensive details regarding development of the larger survey and details on survey respondents are reported elsewhere. ${ }^{5}$

\section{Participants}

A total of 844 SLPs started the survey, with 575 completing all questions. Participants reported having caseloads that ranged from 8 to 146 children, with a mean of 51. Participants reported working as an SLP from 0 to $20+$ years. The majority of participants (70\%) had less than 20 years of experience.

\section{Survey Questions}

To answer our first research question, we examined responses from the survey item: $A t$ your school, which factors are mandatory for determining service eligibility for a child with a speech sound disorder? (Select all that apply). Participants could choose from any of the following: standardized scores, intelligibility ratings, it severely interferes with communication, it attracts adverse attention, it adversely affects educational performance, and other (please specify).

For our second research question, we examined responses to two survey items: (1) How many standardized test scores are required to determine eligibility for services for a child with a speech sound disorder? (2) What standard score is 
necessary for a child with a speech sound disorder to qualify for services?

To answer our third research question, we analyzed responses to two survey items. (1) Do you agree with the eligibility criteria used at your school for children with speech sound disorders? Response options were yes and no with an optional comment box. (2) Based on the eligibility guidelines that you are required to follow, have you had referrals for speech sound production issues for children who you feel should receive services but don't qualify? Response options were yes and no with an optional comment box.

Finally, we examined responses to the survey item: Which items are part of assessment for speech sound disorders at your school? (Select all that apply). Participants chose from any or all of the following options: oral mechanism examination, standardized testing, stimulability testing, informal speech sample, classroom observation, teacher questionnaire, parent questionnaire, receptive and expressive language assessment, literacy assessment, and other (please specify).

\section{Data Analysis}

We did not require participants to answer all questions in the survey. As such, some respondents answered only some of the questions and/ or exited the survey without completing all questions. For those respondents, we included their data as it related to our current research questions. Note, however, that those respondents may not have completed the entire survey. The data were analyzed using descriptive sta- tistics. SPSS version 25.0 was used for all analyses.

\section{RESULTS}

\section{Factors and Criteria for Determining Eligibility}

We began our data analysis by looking at responses from the following item on the questionnaire: "At your school, which factors are mandatory for determining service eligibility for a child with a SSD?" Participants could select multiple items from the following list: (1) standardized scores, (2) intelligibility ratings, (3) it severely interferes with communication, (4) it attracts adverse attention, (5) it adversely affects educational performance, and (6) other (please specify). We calculated the percentage of total respondents who selected each variable. Fig. 1 shows the percentage of SLPs who chose each item. "It adversely affects educational performance" and "standardized tests" had the highest percentage of responses (86.6 and 62.6\%, respectively) and "It attracts adverse attention" had the lowest percentage of responses (23.1\%), aside from "other" (14.7\%). Next, we calculated the sum of items chosen for each respondent. On average, participants reported 2.7 items were mandatory for determining service eligibility, with a range of 0 to 6 .

Our second research question sought to explore more specific details regarding how standard scores are used for eligibility. As such, we examined descriptive information

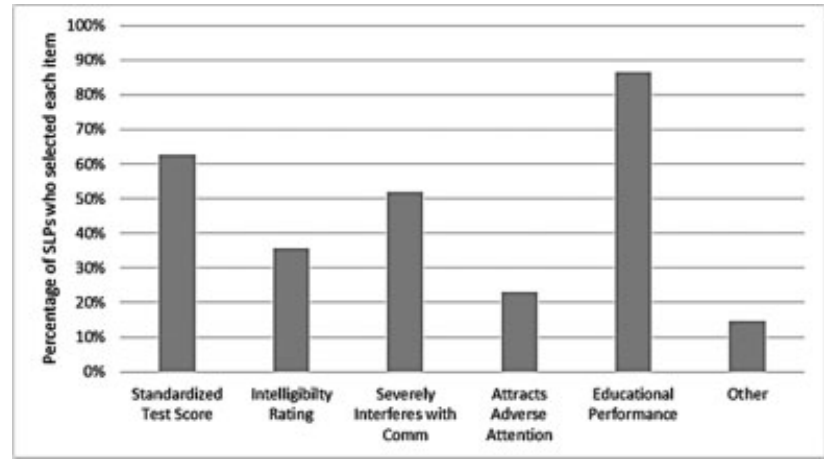

Figure 1 SLP-reported mandatory eligibility criteria for students with SSD based on all survey responses ( $n=597$ ). The labels on the $x$-axis correspond to individual factors that participants could select (could select multiple). Comm, communication. 
regarding the number of standardized measures and what cut-points are required for eligibility. More than half (65.4\%) of the sample indicated that one standardized test score was adequate to determine eligibility; $39 \%$ of respondents indicated that no specific cut-point (e.g., below 1 standard deviation) was used.

\section{Agreement with Eligibility Criteria}

For our third research question, we were interested in how many school-based SLPs agree with the earlier-reported eligibility criteria. In this sample, $81.5 \%$ of SLPs reported agreeing with their school's eligibility criteria $(n=466)$. Next, we asked participants to reflect if there had been children who they believed should have received services for SSD, but did not receive them because of the mandated eligibility criteria; $37 \%$ of the sample reported "yes."

\section{Assessment Measures Used for Eligibility}

To answer our final question, we analyzed responses to the question, "Which items are part of an assessment for SSDs at your school?" Participants could choose multiple items from this list: (1) oral mechanism exam, (2) standardized testing, (3) stimulability testing, (4) informal speech sample, (5) classroom observation, (6) teacher questionnaire, (7) parent questionnaire, (8) receptive and expressive language assessment, (9) literacy assessment, or (10) other. Fig. 2 shows the percentage of SLPs who chose each item. The mean number of items chosen was $6(S D=1.4$, range $=2-9)$. "Standardized testing" received the highest percentage of responses (73.5\%) and "literacy assessment" received the lowest (3.2\%).

\section{DISCUSSION}

The factors school-based SLPs consider when determining eligibility criteria for children with SSD are constrained by federal guidelines. ${ }^{5-7}$ These parameters broadly outline that eligibility for school-based special education services is contingent upon the presence of a diagnosed disability, the extent to which the disability negatively influences the child's educational performance, and the extent to which specific instructions and services are critical to ensure the child's progress. However, explicit rules and regulations for how to evaluate these criteria may vary at the state and district level. Certainly, school-based SLPs are often directed to utilize a limited set of criteria, and even specific assessment tools to inform those criteria. Thus, the extent to which there is variability within the strictures of eligibility for school-based services is inevitable, yet not well understood. Earlier, we presented some findings from a nationally distributed online survey that described the current state of practice regarding both the eligibility criteria and assessments that are most often used to determine eligibility. In the following paragraphs, we consider the

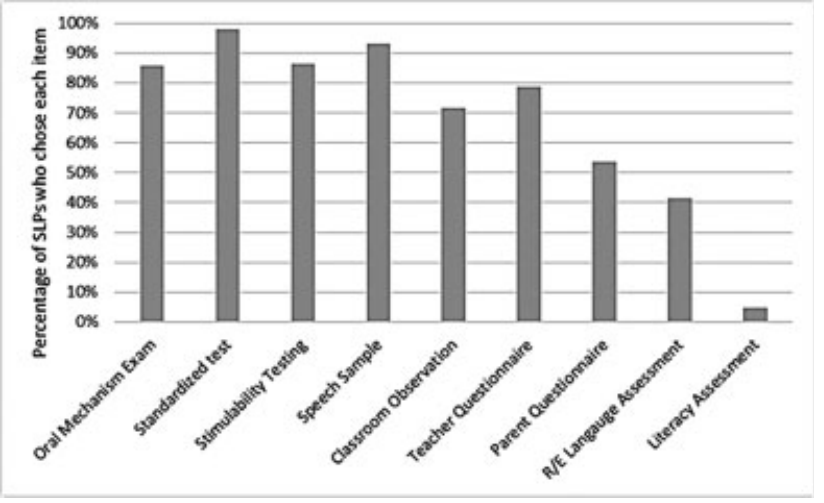

Figure 2 Assessment components that SLP respondents use to evaluate speech sound disorders $(n=578)$. The labels on the $x$-axis correspond to assessment components that participants could select (could select multiple). R/E language assessment, receptive/expressive language assessment. 
implications these results may have on current service provision in the schools.

One of the primary questions on the survey concerned the mandatory criteria that SLPs must use to determine service eligibility for children with SSD. The actual source(s) of the mandated guidelines are described elsewhere ${ }^{5}$ and were quite variable (i.e., state, district, federal). However, an overwhelming majority of survey respondents appeared to adhere to foundational IDEA federal guidelines by selecting "adversely affects educational performance" as one of the mandated eligibility criteria. From a possible six items, participants indicated that on average, two to three items were deemed to be mandatory. A subsequent question posed to participants asked whether they agreed with the prescribed eligibility criteria. Nearly 20\% of SLPs indicated that they did not fully agree with the mandatory criteria. This finding was compounded by the fact that over one-third of SLPs reported that they had previously evaluated a child who they believed requires services, but did not meet the state guidelines.

It is beyond the scope of this work to extrapolate the reasons for disagreement between the directives SLPs must adhere to and what they regard as "best practice." However, these results do underscore a clear and unfortunate disconnect between training, practice, and policy that must be better understood and addressed at the state level, if not federally. Congruence between the governing association for SLPs (American Speech-Language-Hearing Association; ASHA) and federal law is critical for consistent training and education programs. This may be particularly true for children with SSD, who constitute a heterogeneous group both in terms of the nature and severity of their disorder.

In general, the heterogeneity observed in children with SSD is reflected in the array of assessments and treatments that SLPs reportedly utilize,,$^{2,17,21-23}$ and results discussed earlier clearly align with prior studies in this regard. Skahan et $\mathrm{al}^{17}$ surveyed 333 school-based SLPs on their assessment practices; although over $70 \%$ reported using a measure of intelligibility as well as a standardized articulation test, many reported using a variety of direct assessment procedures. Results from the present study suggest that these particular measures (standardized tests and speech samples) remain among the most utilized assessment methods for children with SSD in current practice. Our results also confirm that SLPs continue to use many different types of assessments, with reporting SLPs indicating they use six different assessments, on average. While on the one hand, this variability may permit flexibility in how SLPs evaluate eligibility criteria, this continued inconsistency may also reflect a critical lack of information about which types of assessments are most efficient and effective. Indeed, future research, particularly practice-based evidence, is critical for supporting school-based service provision that is both practical and effectual.

Unfortunately, data from the present survey also indicate that despite growing evidence that school-age children with SSD are at increased risk for subsequent reading difficulties, ${ }^{15,16,24,25}$ only $5 \%$ of SLPs reported using a literacy assessment. This proportion is considerably less than the $12 \%$ of SLPs in Skahan et al's ${ }^{17}$ study who reported routinely incorporating a phonemic awareness assessment for their students with SSD. The present results are somewhat surprising, as almost $90 \%$ of SLPs indicated that mandated eligibility criteria included an observed adverse effect on the child's educational performance. A literacy assessment would certainly provide strong evidence as to whether a child's diagnosis was negatively impacting their educational attainment. However, descriptive studies of school-based practices ${ }^{24}$ do suggest that very few SLPs actively engage in literacyfocused therapy activities, even when literacy is a therapeutic goal. Results from the present study, taken together with research from the past decade, suggest that school-based clinical practice has perhaps not shifted sufficiently to meaningfully address the literacy risks that many school-age children with SSD may face.

\section{CONCLUSION}

To summarize, we sought to describe and understand the variability that currently exists with respect to eligibility criteria for schoolbased service provision to children with SSD. Children with SSD exhibit a range of difficulties, and developing appropriate and 
standardized treatment expectations is complex and challenging. This challenge is reinforced by the range of eligibility criteria that school-based SLPs must adhere to across the nation, and by the assortment of assessment tools SLPs use to evaluate eligibility criteria. Although variability might be acceptable to some degree, as it allows for flexibility to meet specific statewide or district-based needs, the fact that unexpected differences exist needs to be better understood. Moreover, the fact that a relatively significant proportion of SLPs do not fundamentally agree with mandated criteria must be addressed. Finally, despite consistent study findings that children with SSD are at risk for literacy difficulties, current policy and practice geared toward reducing those difficulties are not sufficient. It is hoped that this description of current practice will encourage further research in these areas and substantiate a reconsideration and revision of policies concerning mandatory eligibility criteria and appropriate assessments for providing balanced and well-rounded school-based services for children with SSD.

\section{DISCLOSURES}

The authors have no financial or nonfinancial relationships to disclose.

\section{CONFLICT OF INTEREST}

None.

\section{REFERENCES}

1. Ireland M, Conrad BJ. Evaluation and eligibility for speech-language services in schools. Perspect ASHA Spec Interest Groups 2016;1(16):78-90

2. Kamhi AG. Treatment decisions for children with speech-sound disorders. Lang Speech Hear Serv Sch 2006;37(04):271-279

3. Swaminathan D, Farquharson K. Using response to intervention for speech sound disorders: exploring practice characteristics and geographical differences. Perspect ASHA Spec Interest Groups 2018;3 (16):53-66

4. Farquharson K, Tambyraja SR, Justice LM, Redle EE. IEP goals for school-age children with speech sound disorders. J Commun Disord 2014;52:184-195

5. Farquharson K, Boldini L. Variability in interpreting educational performance for children with speech sound disorders. Lang Speech Hear Serv Sch 2018;49(04):938-949

6. US Department of Education. Twenty-Fourth Annual Report to Congress on the Implementation of the Individuals with Disabilities Education Act. 2004

7. Thomas JL. Decoding eligibility under the IDEA: interpretations of "adversely affect educational performance.”. Campbell Law Rev 2016;38(01): 73-107

8. Hayiou-Thomas ME, Carroll JM, Leavett R, Hulme C, Snowling MJ. When does speech sound disorder matter for literacy? The role of disordered speech errors, co-occurring language impairment and family risk of dyslexia. J Child Psychol Psychiatry 2017;58(02):197-205

9. Hitchcock ER, Harel D, Byun TM. Social, emotional, and academic impact of residual speech errors in school-age children: a survey study. Semin Speech Lang 2015;36(04):283-294

10. Lewis BA, Freebairn LA, Taylor HG. Correlates of spelling abilities in children with early speech sound disorders. Read Writ 2002;15(3-4):389-407

11. Lewis BA, et al. Heritability and longitudinal outcomes of spelling skills in individuals with histories of early speech and language disorders. Learn Individ Differ 2018;65:1-11

12. Overby MS, Trainin G, Smit AB, Bernthal JE, Nelson R. Preliteracy speech sound production skill and later literacy outcomes: a study using the Templin Archive. Lang Speech Hear Serv Sch 2012;43(01):97-115

13. Preston JL, Edwards ML. Phonological processing skills of adolescents with residual speech sound errors. Lang Speech Hear Serv Sch 2007;38(04): 297-308

14. Preston JL, Hull M, Edwards ML. Preschool speech error patterns predict articulation and phonological awareness outcomes in children with histories of speech sound disorders. Am J Speech Lang Pathol 2013;22(02):173-184

15. Raitano NA, Pennington BF, Tunick RA, Boada $\mathrm{R}$, Shriberg LD. Pre-literacy skills of subgroups of children with speech sound disorders. J Child Psychol Psychiatry 2004;45(04):821-835

16. Cabbage KL, Farquharson K, Iuzzini-Seigel J, Zuk J, Hogan TP. Exploring the overlap between dyslexia and speech sound production deficits. Lang Speech Hear Serv Sch 2018;49(04): 774-786

17. Skahan SM, Watson M, Lof GL. Speech-language pathologists' assessment practices for children with suspected speech sound disorders: results of a national survey. Am J Speech Lang Pathol 2007; 16(03):246-259

18. Spaulding TJ, Plante E, Farinella KA. Eligibility criteria for language impairment: is the low end of 
normal always appropriate? Lang Speech Hear Serv Sch 2006;37(01):61-72

19. Macrae T. Stimulus characteristics of single-word tests of children's speech sound production. Lang Speech Hear Serv Sch 2017;48(04):219-233

20. McLeod S, Verdon S. A review of 30 speech assessments in 19 languages other than English. Am J Speech Lang Pathol 2014;23(04): 708-723

21. Farquharson K, Tambyraja SR, Schmitt MB, Justice LM. Describing school-based therapy for children with speech sound disorders with and without concomitant language impairment. Poster session presented at the Annual Convention of the American Speech-Language-Hearing Association; November 2016; Philadelphia, PA
22. Joffe V, Pring T. Children with phonological problems: a survey of clinical practice. Int J Lang Commun Disord 2008;43(02):154-164

23. Farquharson K, Hogan TP, Bernthal JE. Working memory in school-age children with and without a persistent speech sound disorder. Int J SpeechLanguage Pathol 2018;20(04):422-433

24. Tambyraja SR, Schmitt MB, Justice LM, Logan JA, Schwarz S. Integration of literacy into speechlanguage therapy: a descriptive analysis of treatment practices. J Commun Disord 2014;47:34-46

25. Johnson CJ, Beitchman JH, Brownlie EB. Twentyyear follow-up of children with and without speech-language impairments: family, educational, occupational, and quality of life outcomes. Am J Speech Lang Pathol 2010;19(01):51-65 